\title{
The Distribution of Grain Boundary Planes in Interstitial Free Steel
}

\begin{abstract}
HOSSEIN BELADI and GREGORY S. ROHRER
The grain boundary character distribution in a commercial IF steel has been measured as a function of lattice misorientation and boundary plane orientation. The grain boundary plane distribution revealed a relatively low anisotropy with a tendency for grain boundaries to terminate on low index planes having relatively low surface energy and large interplanar spacings. Although the most common grain boundary plane orientation was (111), grain boundaries terminated on higher index planes were sometimes found. For instance, at a misorientation angle of 60 deg about [111], symmetric $\{112\}$ tilt boundaries were far more populous than [111] twist boundaries. The current observation revealed an inverse relationship between the measured populations and the previously reported grain boundary energies.
\end{abstract}

DOI: $10.1007 / \mathrm{s} 11661-012-1393-0$

(C) The Minerals, Metals \& Materials Society and ASM International 2012

\section{INTRODUCTION}

INTERSTITIAL free (IF) steels are widely employed for automobile panels as they offer excellent deep drawability. This property is mainly attributed to the control of a unique texture development (i.e., $\gamma$-fiber) through the recrystallization process. ${ }^{[1]}$ Surprisingly, less attention has been given to the role of grain boundary characteristics to the outstanding property in IF-steels, though the presence of grain boundaries in polycrystalline materials significantly controls their mechanical behavior.

The grain boundaries can, for instance, act as the source of dislocations and the resistance to the dislocation motion from one grain to another. This results in an increase in the strength with a decrease in the grain size (i.e., dislocation mean free path) as illustrated by the Hall-Petch relationship. ${ }^{[2,3]}$ Interestingly, ferritic steels generally exhibit an abnormally high Hall-Petch slope (i.e., typically on the order of $20 \mathrm{MPa} / \mathrm{mm}^{0.5}$ ) compared with other metals (e.g., austenitic steels). This is primarily linked to the presence of interstitial elements (i.e., carbon) at the grain boundaries. ${ }^{[4]}$ However, a similar trend was reported for IF steels ${ }^{[5]}$ where the interstitial elements are negligible. This encourages investigation of the characteristics of the grain boundary plane (i.e., distribution and energy) in IF steels; because the grain boundaries are active structural elements, certain properties of crystalline materials are controlled to a large extent by their characteristics.

Five grain boundary parameters are required to quantitatively characterize the grain boundary plane

HOSSEIN BELADI, Senior Research Academic, is with Institute for Frontier Materials, Deakin University, Geelong, VIC 3216, Australia. Contact e-mail: hossein.beladi@deakin.edu.au GREGORY S. ROHRER, W.W. Mullins Professor and Head, is with Department of Materials Science and Engineering, Carnegie Mellon University, Pittsburgh, PA 15213-3890.

Manuscript submitted June 17, 2012.

Article published online September 8, 2012 distribution, consisting of three misorientation parameters to describe the orientation relationship across the grain boundary, and two parameters specifying the orientation of the boundary plane. ${ }^{[6]}$ Although, the former can be readily obtained through conventional two-dimensional electron back-scattered diffraction (EBSD), the latter, indeed, needs new tools such as the dual beam focused ion beam scanning electron microscope (i.e., serial sectioning) and high energy X-ray tomography, to precisely resolve and visualize the threedimensional internal microstructures of materials. However, limited work has been undertaken to date for the grain boundary characterization using advanced threedimensional analysis techniques ${ }^{[7,8]}$ due to their complexity and time constraints.

Recently, all five grain boundary parameters can statistically be measured through the conventional EBSD orientation mapping technique. This unique procedure, which is described in detail elsewhere, ${ }^{[6]}$ enables us to successfully measure the grain boundary plane orientation distribution along with the misorientation distribution. This technique was employed to survey the grain boundary plane distribution in different materials, such as $\mathrm{Al}^{\left[{ }^{[9]}\right.} \mathrm{SrTiO}_{3},{ }^{[10]} \mathrm{TiO}_{2},{ }^{[11]} \mathrm{MgAl}_{2} \mathrm{O}_{4},{ }^{[11]}$ $\mathrm{MgO}^{[12]}$ and determine the correlation between the grain boundary plane distribution and the grain boundary energy. These measurements generally revealed remarkable anisotropy in the distribution of grain boundary planes at a given lattice misorientation. Furthermore, the distribution of grain boundary surfaces was widely dominated by low index interfaces, which indeed appeared as low energy surface planes. ${ }^{[12]}$

The five-parameter analysis has been widely employed for ceramic materials and face-centered cubic metals. The only five-parameter analysis on body-centred cubic crystal structure was a very limited study of the grain boundary distribution of a Fe-1 pet Si alloy. ${ }^{[13]}$ In view of the continuing interest in the distribution of grain boundary planes, and our limited knowledge of this distribution in technologically important ferritic steels, 
the objective of the present paper is to provide a detailed description of the grain boundary character distribution in a commercial IF steel using the five-parameter grain boundary analysis technique. The experimental observations are compared with calculated grain boundary energies $^{[14]}$ to examine whether the relationship between the boundary plane distribution and energy reported for other materials is maintained in a body-centered cubic metal (i.e., IF steel).

\section{EXPERIMENTAL PROCEDURE}

The IF steel composition used in this study was $0.003 \mathrm{C}-0.13 \mathrm{Mn}-0.08 \mathrm{Ti}-0.03 \mathrm{Al}-0.004 \mathrm{~N}$ (wt pct). The experimental material was in the form of slab product with a thickness of $25 \mathrm{~mm}$. The thickness of material was initially reduced to $5 \mathrm{~mm}$ through multipass rolling in the temperature range of $1473 \mathrm{~K}$ to $1273 \mathrm{~K}\left(1200{ }^{\circ} \mathrm{C}\right.$ to $1000{ }^{\circ} \mathrm{C}$ ) followed by air cooling. The material was then cold-rolled to 80 pct reduction and annealed at $1073 \mathrm{~K}\left(800{ }^{\circ} \mathrm{C}\right)$ for $15 \mathrm{~min}$ in a fluid bed furnace in flowing nitrogen gas.

Samples for EBSD were prepared by standard mechanical polishing, finished with a colloidal silica slurry polish. EBSD measurements were carried out using a FEGSEM Quanta 3D FEI scanning electron microscope operated at $20 \mathrm{kV}$. The instrument was equipped with a fully automated EBSD device attachment. Data acquisition and post processing were performed using the TexSEM Laboratories, Inc. software (TSL). Multiple EBSD maps were acquired using a spatial step size of $1 \mu \mathrm{m}$ on a hexagonal grid. The total area covered approximately $26 \mathrm{~mm}^{2}$ containing more than 50,000 distinct grains. The average confidence index generally varied between 0.80 and 0.85 .

A grain dilation clean-up function in the TSL software was employed for all orientation maps to remove ambiguous data. A single orientation was then assigned to a given grain through averaging all orientation data belonging to that grain. Boundary line traces/segments were extracted after smoothing of uneven grain boundaries using the reconstruction grain boundary function in TSL software and employing a boundary deviation limit of 2 pixels (i.e., $2 \mu \mathrm{m}$ ). There were $\sim 300,000$ line traces after excluding the boundary segments of less than $3 \mu \mathrm{m}$. These line traces were employed to calculate the five-parameter grain boundary character distribution using a procedure described in detail elsewhere. ${ }^{[6]}$

The stereological calculation of the grain boundary character distribution assumes that the grain pairs are uniformly distributed in orientation space. In other words, each collection of bicrystals in the data set with the same misorientation must be randomly orientated. However, in the present case, where the sample is textured, this assumption does not apply. To account for the texture, we calculated the grain boundary character distribution using a procedure that removes the textureinduced bias in the distribution of observed bicrystal orientations. Specifically, all observations associated with a specific misorientation type were weighted inversely according to their frequency of appearance in a given range of orientation space. This procedure recovers the desired random distribution of orientations for each bicrystal type and removes texture induced bias in the stereological calculation. The analysis was carried out at 11 bins per 90 degree level of discretization, which offers about 8.2 degree resolution (i.e., 85 pct of the bins contained at least five observations) in the current study. The grain boundary character was shown in stereographic projection and presented as multiples of a random distribution (MRD) corresponding to relative areas (i.e., values greater than one indicate planes that are observed more frequently than expected in a random distribution).

\section{RESULTS}

The annealed microstructure of IF steel consisted of equiaxed grains with an average grain size of $13 \mu \mathrm{m}$ (Figure 1). The material revealed a pronounced $\gamma$-fiber $\langle 111\rangle|| N D$ crystallographic texture (Figure 2(a)), which is typical for cold rolled and annealed IF steels. ${ }^{[1]}$ The inverse pole figure was characterized by a relatively strong texture having a maximum of 8.8 times random intensity (Figure 2(b)), with stronger (111) fibers oriented along the normal direction. The presence of the fiber texture resulted in a relatively uniform grain boundary misorientation angle distribution, which is quantitatively different from randomly textured materials (i.e., random misorientation angle distribution, ${ }^{[15]}$ also known as Mackenzie distribution) having a maxima at about $45 \mathrm{deg}$ misorientation angle. It is rather a bimodal distribution revealing two peaks at low (i.e., $\sim 10 \mathrm{deg}$ ) and high (i.e., $~ 50$ ) misorientation angles (Figure 3(a)). This is also unlike the misorientation angle distribution in fcc metals, where the highest population of boundaries is centered about the $60 \mathrm{deg}$ misorientation angle. ${ }^{[16]}$ Similarly, it appeared that the

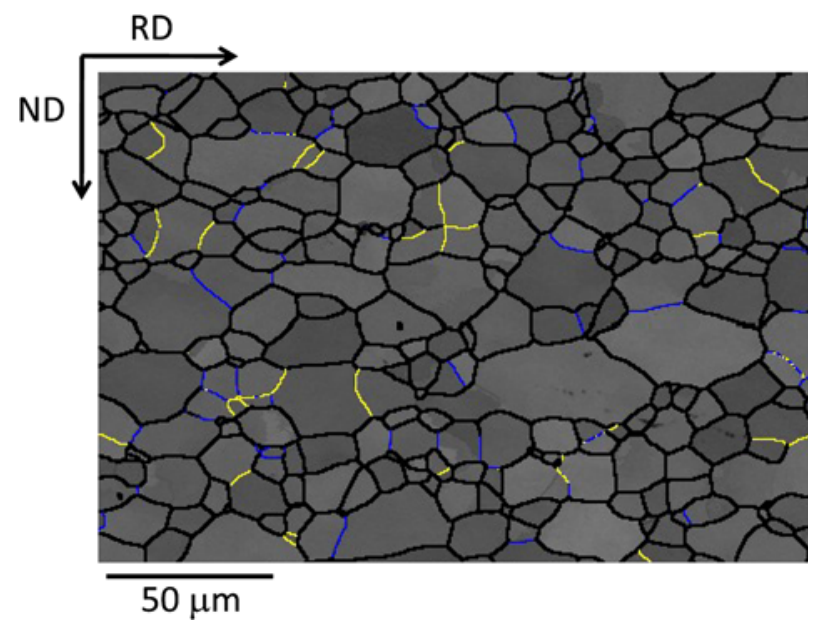

Fig. 1-EBSD map of IF steel microstructure after 80 pct cold rolled followed by reheating at $1073 \mathrm{~K}\left(800{ }^{\circ} \mathrm{C}\right)$ for $15 \mathrm{~min}$. Yellow, blue and black lines represent boundaries with misorientation angles $(\theta)$ of $5 \mathrm{deg}<\theta<10 \mathrm{deg} ; 10 \mathrm{deg}<\theta<15 \mathrm{deg}$, and $\theta<15 \mathrm{deg}$, respectively. RD and ND are rolling and normal directions, respectively (Color figure online). 

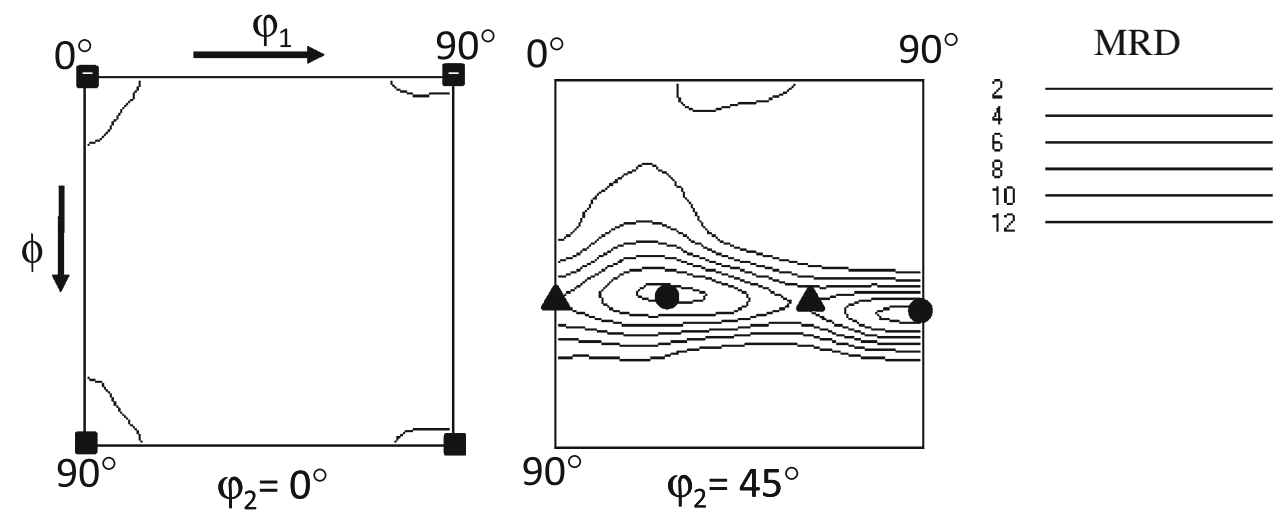

(a)

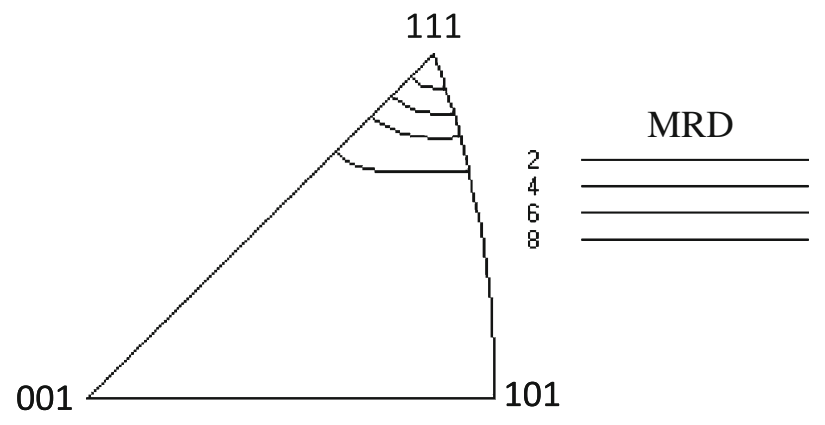

(b)

Fig. 2-(a) Orientation distribution function of the IF steel at the cold rolled and annealing condition. $\{001\}\langle 110\rangle$; $\{111\}\langle 112\rangle$ $\boldsymbol{\Delta}\{111\}\langle 110\rangle$. (b) Inverse pole figure of the cold rolled and annealed IF steel along the normal direction. MRD is multiples of a random distribution.

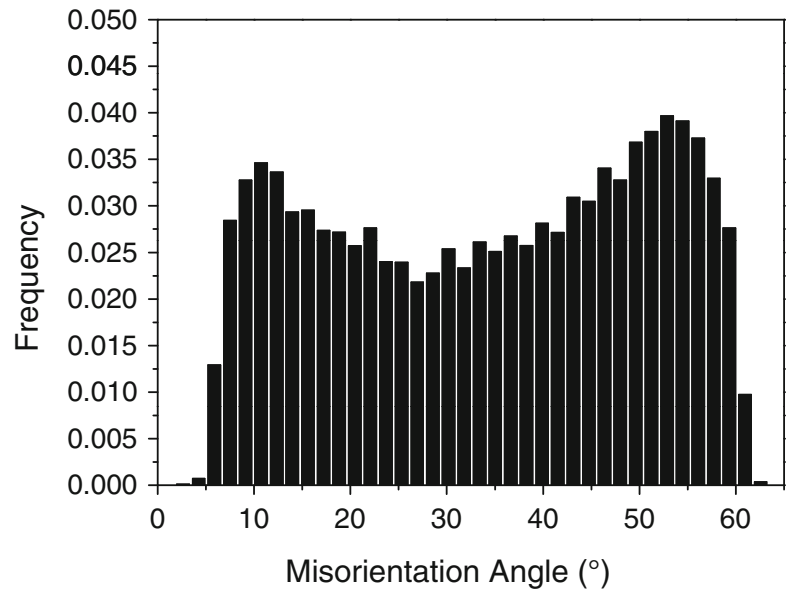

(a)

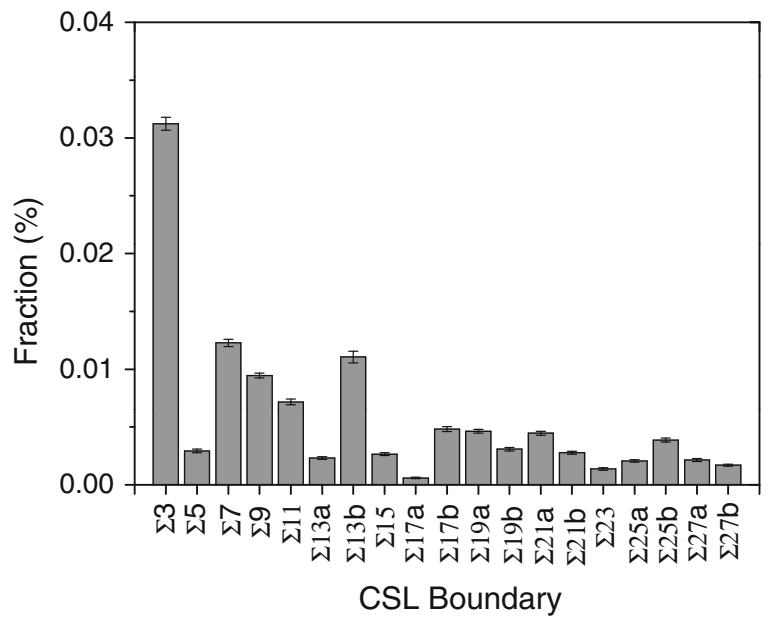

(b)

Fig. 3-Misorientation angle distribution (a) and CSL boundaries fractions $(b)$ of the cold rolled and annealed IF steel.

population of the coincidence site lattice (CSL) boundaries was significantly low compared with fcc materials (e.g., $\mathrm{Ni}$ and $\mathrm{Cu}$ in Reference 17), though the $\Sigma 3$ boundary revealed a relatively greater population among others (Figure 3(b)). The CSL boundaries were classified using Brandon criterion, $\Sigma 3^{n}$ with $n \geq 3$ (i.e., $n=3,5,7, \ldots$,up to 27 ), as a function of total boundary length.
The parameterization of the distribution in Figure 3 reflects only the misorientation angle. To also show the distribution of misorientation axes, $\lambda(\Delta g)$, averaged over all boundaries planes, Rodrigues-Frank space was employed (Figure 4). The Rodrigues vector $(\Delta g)$ is a unique vector having a direction parallel to the common axis of misorientation and its magnitude is proportional to the tangent of one half of the misorientation angle. 


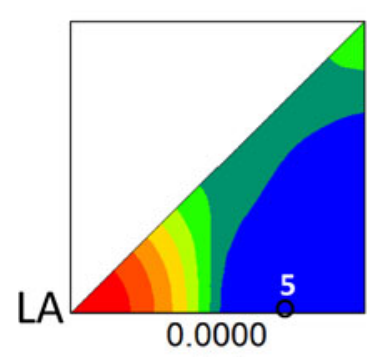

(a)

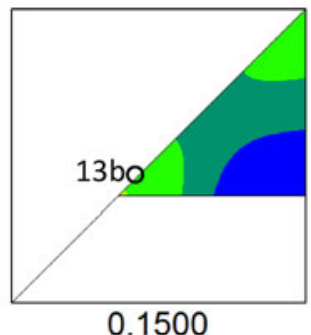

(d)

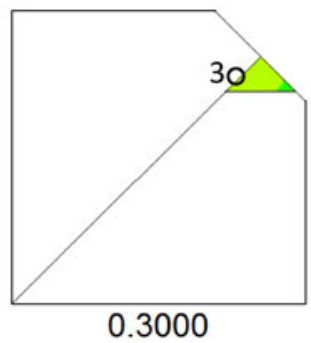

(g)

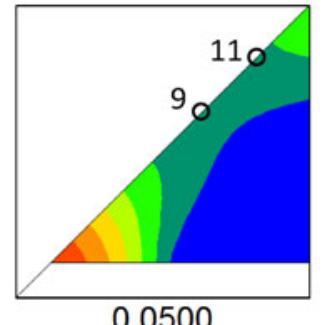

(b)

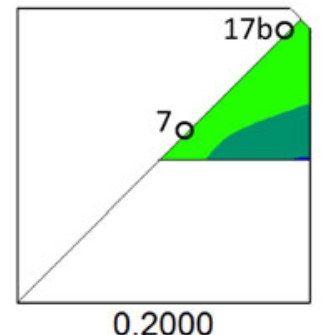

(e)

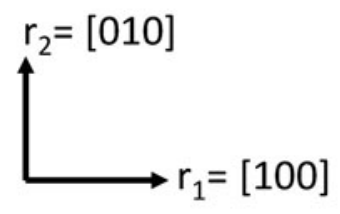$$
\text { g) }
$$

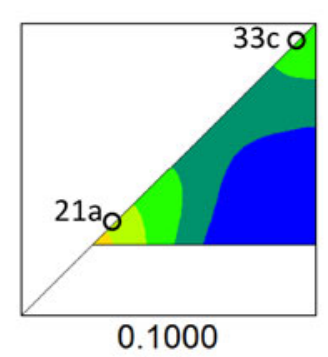

(c)

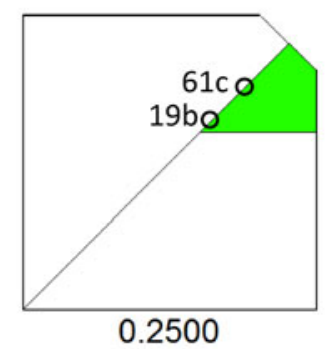

(f)

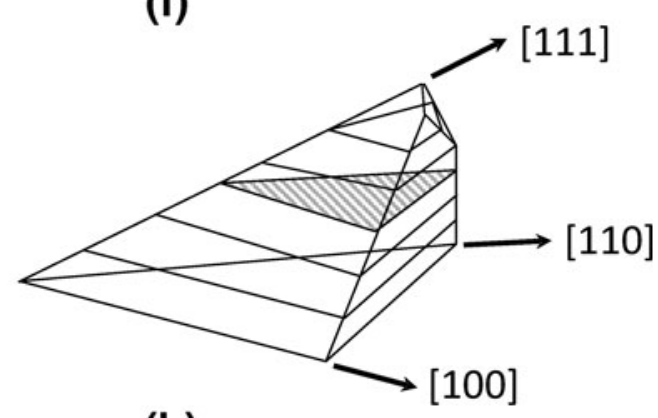

(h)

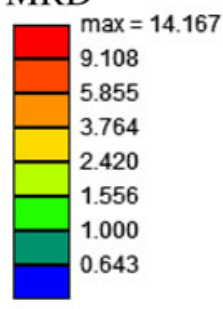

Fig. 4-The distribution of grain boundary misorientations in the three-dimensional fundamental zone in Rodrigues-Frank space for a cold rolled and annealed IF steel. (a) Projection of all data on to the base of the R-F space. (b) through $(g)$ Planar sections perpendicular to the [001] axis $\left(\right.$ i.e., $r_{3}$ ) through the fundamental zone in R-F space $(h)$. The vertical coordinate value is below each section. The position of some CSL boundaries were shown on the edge of sections. LA represents low angle misorientations. MRD is multiples of a random distribution.

The direction of the Rodrigues vector components, $r_{1}, r_{2}$ and $r_{3}$, are parallel to [100], [010] and [001] crystal axes, respectively. The position of misorientations about the [100], [110] and [111] axes are indicated in the fundamental zone of Rodrigues-Frank space shown in Figure 4(h). The projection of misorientation data was presented at different sections through the three dimensional space perpendicular to $r_{3}$ (i.e., [001], Figure 4). The misorientation distribution data revealed significant texture; the distribution has a maximum at low misorientation angles (i.e., 14 times random at maximum). The distribution can also indicate the presence of coincidence lattice boundaries as they can mainly be found on the edges of the Rodrigues-Frank space, as pointed out in Figure 4. The current result revealed the presence of low sigma CSL boundaries (i.e., $\Sigma 3$ ) and some high sigma boundaries such as $\Sigma 61 \mathrm{c}, \Sigma 33 \mathrm{c}$ (Figure 4).

As the Rodrigues-Frank space only represents three parameters of the grain boundary character distribution (i.e., misorientation distribution), the relative area distribution of grain boundary planes, $\lambda(n)$, was plotted in the stereographic projection to investigate the distribution of grain boundary planes in the crystal reference frame. Here, the [001] crystal axis was located perpendicular to the paper plane and the [100] direction pointed horizontally in the plane of the paper to the right. The distribution of grain boundary planes for all misorientations revealed a relatively low anisotropy, having a maximum at the (111) position with a value of 1.36 MRD (i.e., 36 pct greater than expected in a random distribution). The minimum of the distribution was centered at (100) with 0.8 MRD (Figure 5). The distribution was $\sim 1.1$ MRD at the (101) position.

The complete grain boundary character distribution depends on five parameters and this can only be shown by plotting the distribution of grain boundary planes at a fixed misorientation, $\lambda(n \mid \omega /[u v w])$. The grain boundary plane distribution was plotted for specific misorientations about [111], [110] and [100], mainly examining coincident site lattice boundaries (Figures 6, 8, and 9). A schematic reference frame for the projection is plotted for each misorientation axis to highlight the positions of the plane normals associated to the twist boundaries (i.e., parallel to a given misorientation axis) and the tilt boundaries (i.e., perpendicular to the misorientation 
axis, Figures 6(a), 8(a), and 9(a)). The latter, indeed, consisted of symmetric tilt boundaries, where the surfaces on either side of the boundary are the same (e.g., $\{112\} \|\{112\})$ and asymmetric tilt boundaries (i.e. boundary surfaces have different plane indices). We note here that throughout our description of the grain

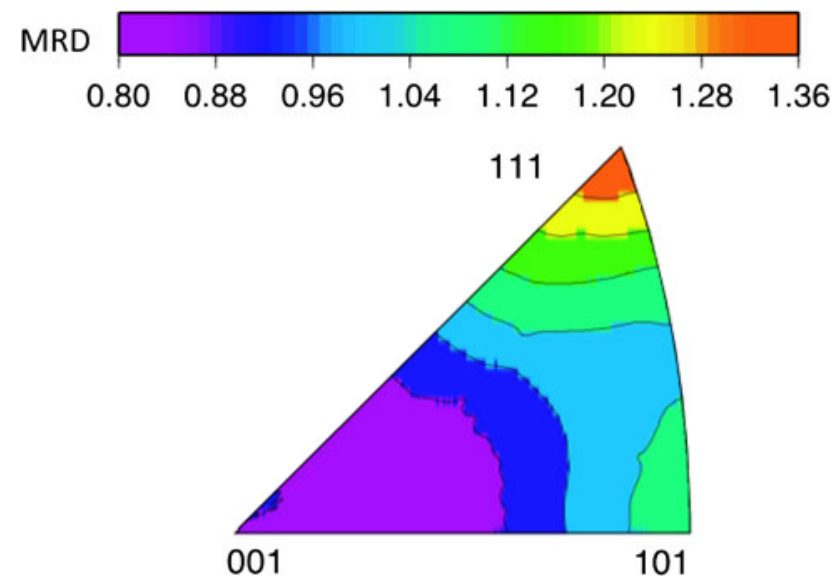

Fig. 5-The distribution of grain boundary planes of the cold rolled annealed IF steel. MRD is multiples of a random distribution. boundary distribution, reference to tilt and twist types apply only to the representation of boundaries in the fundamental zone. As has been pointed out recently by Morawiec, ${ }^{[18]}$ different representations of a single boundary can be classified as tilt, twist, or mixed.

There are significant changes in the distribution of grain boundary planes as a function of misorientation angle for all misorientation axes. At low misorientation angle, the distribution showed peaks on both (111) pure twist and tilt boundaries (Figure 6(b)). At higher misorientation angles about [111], the peak distributions were remarkably altered. The distribution was mainly centered at the (111) twist position, revealing a lower population along the zone of tilt boundaries at a misorientation angle of $22 \mathrm{deg}$ (i.e., $\Sigma 21 \mathrm{a}$, Figure 6(c)). The populations of both tilt and twist boundaries further reduced as the misorientation angle reached 38 deg (i.e., $\Sigma 7$, Figure 6(d)). Beyond that, the population of tilt boundaries gradually increased with the misorientation angle at the expense of the (111) twist boundaries (Figures 6(e), (f) and 7).

Interestingly, the distribution broadened along the zone of tilt boundaries (i.e., the great circle perpendicular to [111] axis, Figures 6(e) through (f)) with misorientation angle, as multiple peaks appeared beyond a $46.8 \mathrm{deg}$ misorientation. However, there was no noticeable

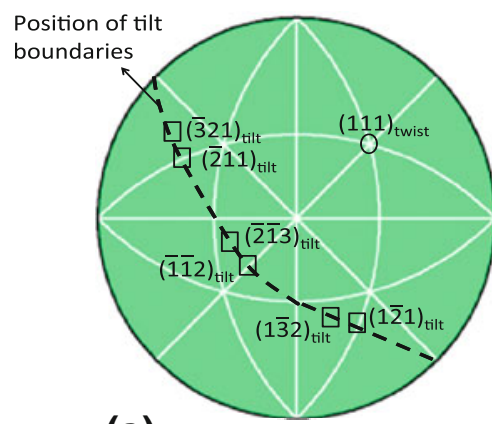

(a)

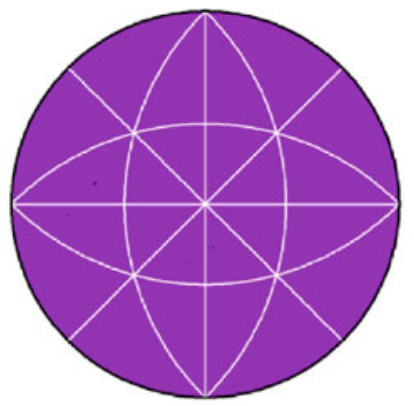

(d) $\sum 7=38.2^{\circ} /[111]$

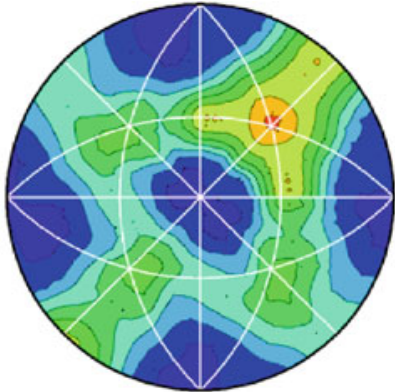

(b) $10 \%[111]$

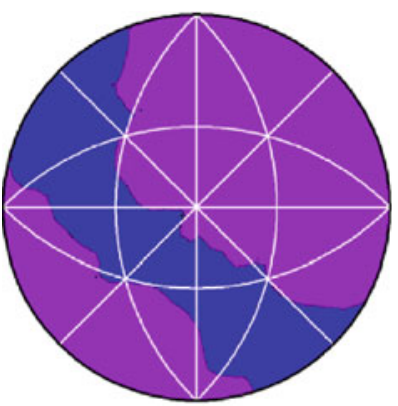

(e) $\sum 19 b=4.8^{\circ} /[111]$

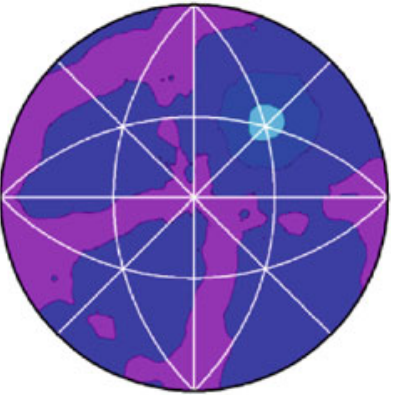

(c) $\sum 21 \mathrm{a}=21.8^{\circ} /[111]$

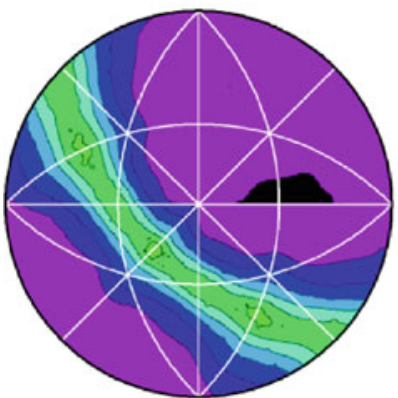

(f) $\sum 3=60^{\circ} /[111]$

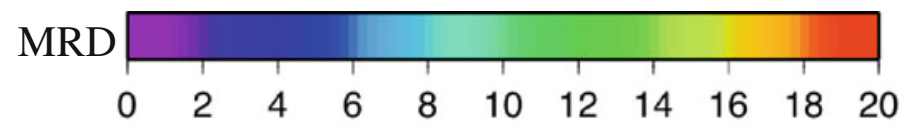

Fig. 6- (a) Schematic representation of the position of the symmetric tilt boundaries (i.e., square marks on the dash line) and the pure twist boundaries (i.e., circle mark) in the grain boundary plane distributions around [111] axis. (b) through ( $f$ ) The distribution of grain boundary plane normals for boundaries with different misorientations around [111] axis: (b) 10 deg, (c) $21.8 \mathrm{deg}$, (d) $38.2 \mathrm{deg}$, (e) $46.8 \mathrm{deg}$, and (f) $60 \mathrm{deg}$. MRD is multiples of a random distribution. 


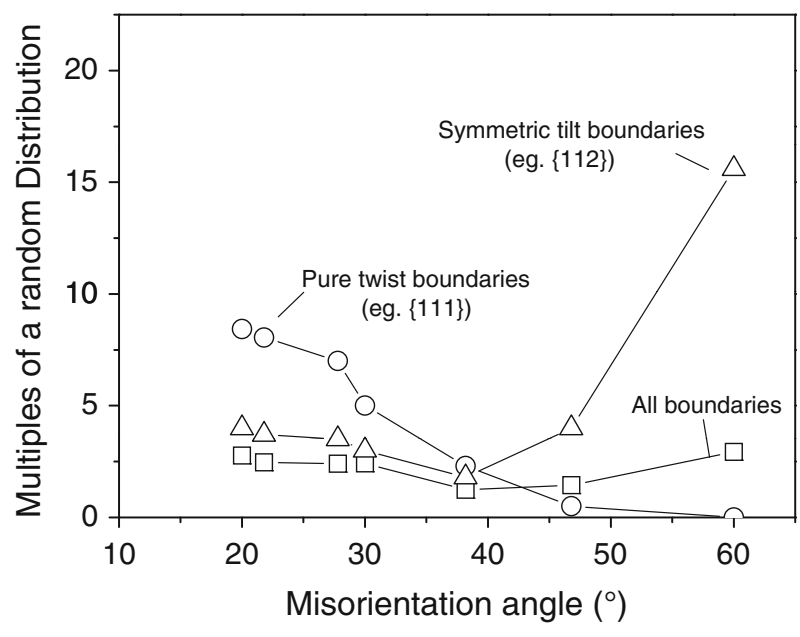

Fig. 7-The multiples of a random distribution of grain boundary plane normals as a function of misorientation angle about [111] axis.

change observed at the position of peak for twist boundary with the misorientation (Figures 6(b) and (c)). These observations can be understood considering the strong preference for given types of planes and the geometric constraints associated with a bicrystal. In a $\{111\}$ pure twist boundary, for example, both crystals can be terminated by a $\{111\}$ plane at the interface. In other words, the plane normals of both adjoining interfaces are parallel. As a result, the distribution of grain boundary planes about the twist positions do not alter noticeably with the change in the misorientation angle. In contrast, the complementary plane in the adjoining crystal to a pure tilt boundary, for example $\{112\}$, would have a different index (i.e., asymmetric tilt boundary). If a crystal is terminated by a (112) plane, the complementary crystal has to be terminated by a $(u v w)$ plane inclined by the misorientation angle away from (112). Consequently, the peak at (112) spreads along the zone axis of the tilt boundaries as the misorientation angle changes. Similar variations in the distribution of grain boundary planes with the misorientation angle were also observed along the zone of tilt boundaries in $\mathrm{MgO}^{[12]}$ and $\mathrm{SrTiO}_{3}{ }^{[10]}$ for the [100] misorientation axis.

At a 60 deg misorientation angle, the distribution maxima were centered at the symmetric tilt boundaries with $16 \mathrm{MRD}$, though the (111) twist boundaries revealed the minimum distribution (i.e., $\sim 0 \mathrm{MRD}$, Figure 6(f)). However, the overall population averaged over all boundary planes at this misorientation is only 2 to $2.5 \mathrm{MRD}$ despite a significant change in the distribution of the maxima (Figure 7). Interestingly, the distribution of grain boundary planes for $\Sigma 3$ boundaries in IF steel revealed almost no presence of (111) $\mid(111)$ 60 deg twist boundaries (so called coherent twin boundaries in fcc metals) and they mostly appeared as symmetric tilt boundaries (e.g., the $\{211\} \|\{211\}$, which are coherent twin planes in bcc crystals ${ }^{[19]}$ ).

A similar trend was observed for the [101] misorientation axis with respect to the misorientation angle (Figure 8). Initially, the population was focused on the
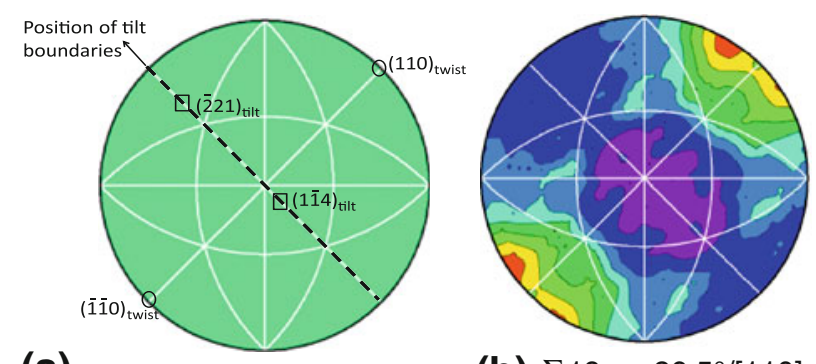

(a)

(b) $\Sigma 19 a=26.5^{\circ} /[110]$
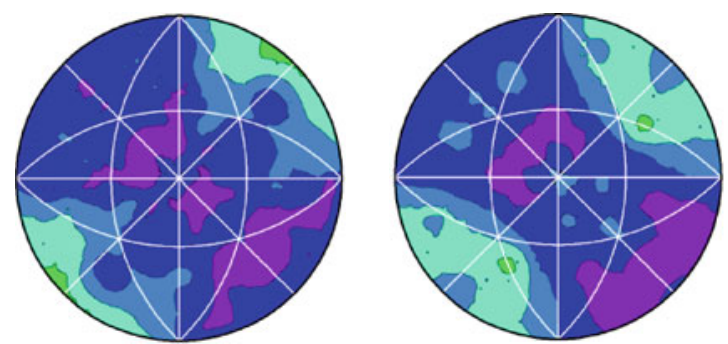

(c) $\Sigma 9=38.9^{\circ} /[110]$

(d) $\Sigma 11=50.5^{\circ} /[110]$

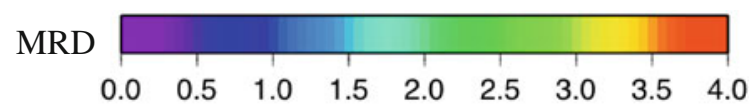

Fig. 8- (a) schematic representation of the position of the symmetric tilt boundaries (i.e., square marks on the dashline) and the pure twist boundaries (i.e., circle mark) in the grain boundary plane distributions around [101]. (b) through $(d)$ The distribution of grain boundary plane normals for boundaries with different misorientations around [101] axis: (b) $26.5 \mathrm{deg}$, (c) $38.9 \mathrm{deg}$, and (d) $50.5 \mathrm{deg}$. MRD is multiples of a random distribution.

$\{110\} \|\{110\}$ twist boundaries (Figure 8(b)). An increase in the misorientation angle gradually resulted in the appearance of the symmetric tilt boundary traces laid on the zone axis of tilt boundaries (i.e., the great circle perpendicular to [110] axis, e.g., $\{221\} \|\{221\}$ in Figures 8(b) through (d)). However, the extent of this change was not as great as the [111] misorientation axis and the lattice misorientation and the twist and tilt boundaries coexisted even at $50.5 \mathrm{deg}$ misorientation (i.e., $\Sigma 11$, Figure 8(d)). In contrast, the distribution for [100] axis was mainly centered on the $\{111\}$ positions at a misorientation angle of $22.6 \mathrm{deg}$ (i.e., $\Sigma 13 \mathrm{a}$, Figure 9(b)). There were also symmetric tilt boundaries, e.g., (012) ||(012), which appeared on the zone axis of the tilt boundaries (i.e., the vertical great circle in the center of stereogram) at low misorientation angle (Figures 9(a) and (b)). As the misorientation angle increased, the population of both $\{111\}$ twist and symmetric tilt (e.g., $\{012\} \|\{012\})$ boundaries significantly decreased and the distribution moved towards the $\{100\}$ twist boundaries (Figures 9(b) through (d)). As a result, the distribution was mainly centered on the $\{100\}$ planes for a misorientation angle of $36.9 \mathrm{deg}$ (i.e., $\Sigma 5$, Figure 9(d)). Finally, it should be noted that the relative population of boundaries with [100] misorientations was significantly less than those with [111] or [110] misorientations.

The population of twist boundaries for all misorientation axes along the edges of the standard stereographic triangle was plotted (Figure 10). The population of twist 
boundaries against twist angle can be observed through horizontal lines for a given misorientation axis. The population intensity was presented in a log scale $(\mathrm{MRD}+1)$. The peaks can be closely correlated to the

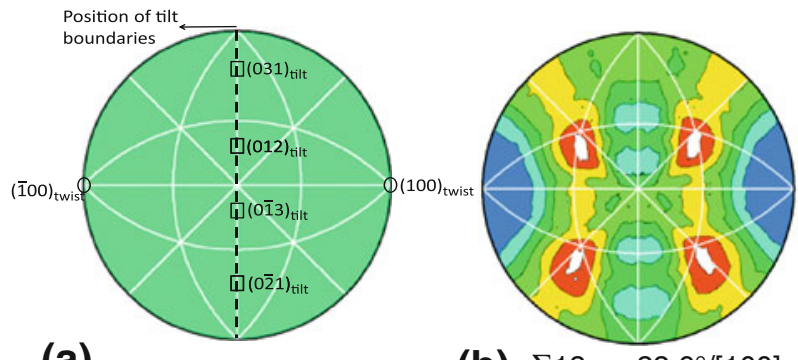

(a)

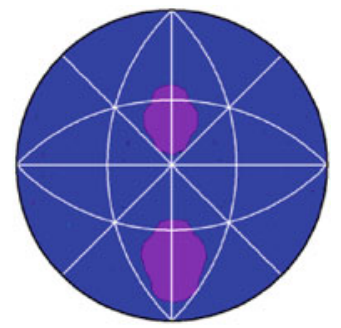

(c) $\Sigma 17 \mathrm{a}=28.1^{\circ} /[100]$

(b) $\Sigma 13 a=22.6^{\circ} /[100]$

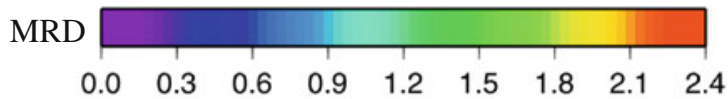

Fig. 9-(a) schematic representation of the position of the symmetric tilt boundaries (i.e., square marks on the dash line) and the pure twist boundaries (i.e., circle mark) in the grain boundary plane distributions around [100]. (b) through $(d)$ the distribution of grain boundary plane normals for boundaries with different misorientations around [100] axis: (b) $22.6 \mathrm{deg}$, (c) $28.1 \mathrm{deg}$, and (d) $36.9 \mathrm{deg}$. MRD is multiples of a random distribution. distribution of grain boundary planes discussed in Figures 6 through 9. The multiple peaks that appeared on the left hand side of the plot corresponded to low misorientation angle boundaries (i.e., $\omega<15$ deg separated by a dash line). Other low misorientation positions were labeled as "LA". The distribution of grain boundary planes for low misorientation angle boundaries cannot be easily explained as they consisted of arrays of dislocations. Generally speaking, the low angle boundaries with the minimum number of dislocations are preferable and they typically are perpendicular to the Burgers vector of the dominant dislocation (i.e., 1/ $2\langle 111\rangle$ for bcc metals $\left.{ }^{[19]}\right)$. There was also a wide peak at twist angles between 15 and $45 \mathrm{deg}$ about the [111] axis, which related to twist boundaries such as $\Sigma 7, \Sigma 19 \mathrm{~b}$ and $\Sigma 21$ a, as observed in Figure 6 . The population of twist boundaries gradually reduced as the misorientation angle approached $60 \mathrm{deg}$ where it reached a minimum. These changes were a result of an increase in the population of tilt boundaries (i.e., symmetric and asymmetric) at the expense of [111] twist boundaries (Figures 6 and 7). There were high populations between [-111] and [-311] at $180 \mathrm{deg}$ and [-311] and [001] at $90 \mathrm{deg}$, which can be referred to an $\{\mathrm{u} 11\}$ twist boundaries. There were also multiple peaks about [110] at misorientation angles of $70 \mathrm{deg}$ and $110 \mathrm{deg}$, which are symmetrically indistinguishable from the misorientations in the fundamental zone and they were very close to $\Sigma 17 \mathrm{~b}$ and $\Sigma 9$ boundaries, respectively. Furthermore, there was a broad local maximum (but still less than $1 \mathrm{MRD}$ ) at a twist angle of $\sim 37 \mathrm{deg}$ for [100] axis, as expected from the distribution of grain boundary planes in Figure 9, which represents $\Sigma 5$. In general, the twist boundaries about [111] and [110] largely revealed higher populations compared with other twist axes.

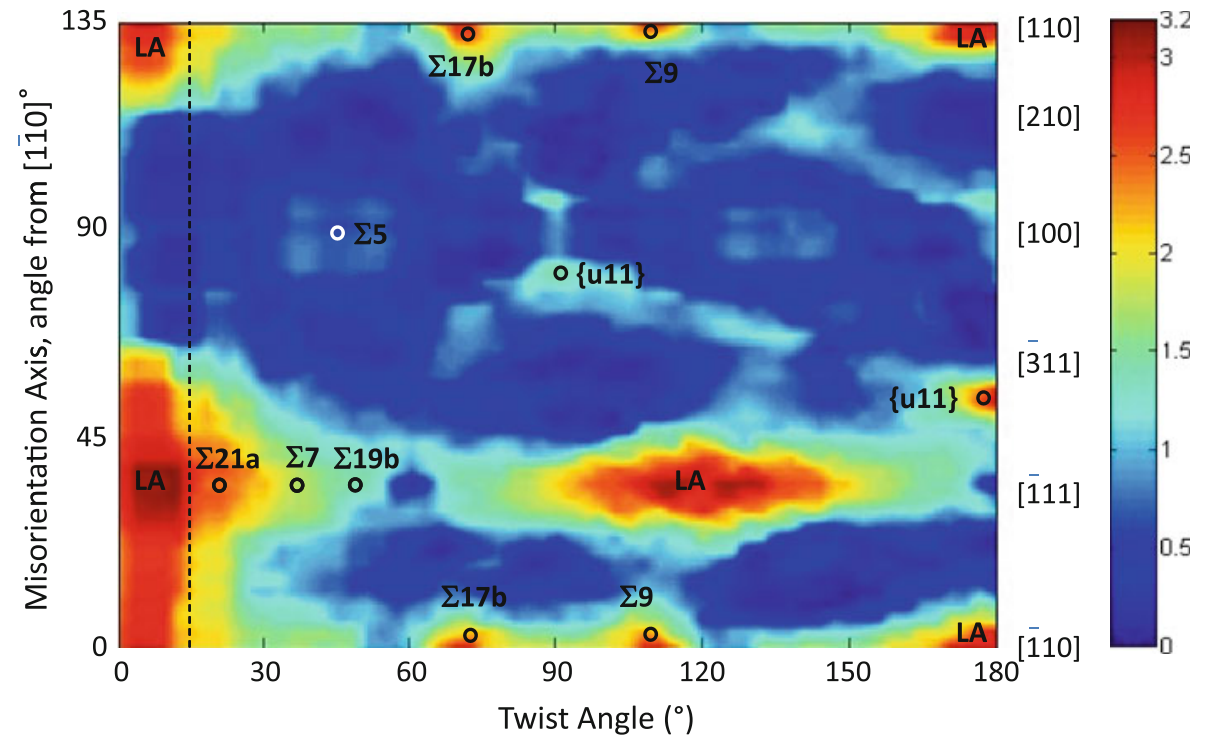

Fig. 10-Twists boundaries for all misorientation axes on the edges of the standard stereographic triangle. LA represents the positions of low misorientations. 


\section{DISCUSSION}

The current five-parameter analysis of IF steel clearly revealed important trends, which cannot be distinguished through the misorientation analysis alone (Figures 3 and 4). The distribution of grain boundary planes averaged over all misorientations highlighted that the internal $\{111\}$ planes occur at about twice the frequency of the $\{100\}$ planes (i.e., the cleavage crystallographic plane in ferritic steels, ${ }^{[20]}$ Figure 5). In other words, the grain boundaries are more frequently terminated by $\{111\}$ planes than any other orientations. However, this is in contrast with the result earlier presented for a Fe-1 pct Si steel, where the minimum frequency was observed on the $\{111\}$ plane and the maximum plane distribution was centered about the $\{110\}$ planes. In addition, very little anisotropy was observed in the distribution of grain boundary planes (i.e., about 10 pct higher than expected for a random distribution). ${ }^{[13]}$

The evolution of microstructure is known to strongly depend on the anisotropy of grain boundary energy. It is known that there is a strong inverse correlation between the grain boundary energy and its population (i.e., the high energy planes have the minimum population and vice versa). ${ }^{[10-12]}$ The energy of grain boundary can closely be correlated with the surface energy as they both imitate the local disruption in atomic bonds at the grain boundary interface. The surface energy anisotropy of $\mathrm{Fe}-3$ pct Si was measured for a temperature range of $1273 \mathrm{~K}$ to $1673 \mathrm{~K}\left(1000{ }^{\circ} \mathrm{C}\right.$ to $\left.1400{ }^{\circ} \mathrm{C}\right)$ using a lattice harmonics series in conjunction with Fourier analysis technique. ${ }^{[21]}$ Interestingly, the temperature revealed a strong effect on the surface energy, as it altered the minimum surface energy for different temperature ranges. The (111) surface had the minimum energy at a temperature between $1473 \mathrm{~K}$ and $1273 \mathrm{~K}\left(1200{ }^{\circ} \mathrm{C}\right.$ and $1000{ }^{\circ} \mathrm{C}$ ), though the minimum surface energy was measured for (110) surface at a temperature range of $1473 \mathrm{~K}$ to $1573 \mathrm{~K}\left(1200{ }^{\circ} \mathrm{C}\right.$ to $\left.1300{ }^{\circ} \mathrm{C}\right)$. Their results also showed a minimum surface energy for (100) at $1673 \mathrm{~K}\left(1400{ }^{\circ} \mathrm{C}\right)$. These changes were discussed in terms of silicon/oxygen absorption on planes at different temperature regimes. This suggests that the current result can be explained through the surface energy measured at $1273 \mathrm{~K}$ to $1473 \mathrm{~K}\left(1000{ }^{\circ} \mathrm{C}\right.$ to $\left.1200{ }^{\circ} \mathrm{C}\right)$, where the (111) revealed the minimum surface energy. In contrast to the Gale et al. ${ }^{[21]}$ observations, Mee $\mathrm{e}^{[22]}$ linked the plane surface energy to the texture development rather than the absorption of oxygen in Fe-3 pct Si. It was also shown that there is enough driving force to promote $(111)\langle u v w\rangle$ texture during the recrystallization at a temperature range of $1373 \mathrm{~K}\left(1100^{\circ} \mathrm{C}\right)$, where (111) surface revealed the minimum energy. ${ }^{[22]}$ Mee's result is consistent with the current observation as there is a pronounced (111)//ND texture due to the static recrystallization and the (111) plane showed a maxima (i.e., most likely due to the minimum surface energy, Figure 5). The difference in the current grain boundary plane distribution with the previous study on $\mathrm{Fe}-1$ pct $\mathrm{Si}^{[13]}$ could, therefore, arise from the differences in the thermomechanical processing or the composition of the alloy.
The current result also confirms that there is a significant anisotropy in the distribution of grain boundary normals at a given lattice misorientation (i.e., $\lambda(n \mid \omega /(u v w)))$ compared with the distribution of internal grain surfaces (i.e., $\lambda(n))$ in IF-steel (Figures 6, 8 , and 9). In addition, the distribution of grain boundary normals for most low CSL boundaries in IF steel is significantly different from those having a fcc structure (e.g., $\mathrm{Cu}, \mathrm{Ni},{ }^{[17]} \mathrm{Al},{ }^{[9]}$ and $\alpha$-brass ${ }^{[23]}$ ). The CSL boundaries observed as pure twist boundaries in fcc metals (e.g., $\Sigma 3=60 /[111]$ ) appeared to be symmetric tilt boundaries in the IF steel (Figure 6(f)) and vice versa (e.g., $\Sigma 5$ and $\Sigma 9$ in Figures 9(d) and 8(c), respectively). This suggests that the distribution of grain boundary normals is significantly changed by the crystal lattice structure for a given misorientation angle and that there is a completely different set of low-energy grain boundary planes in the bcc crystal structure compared with the fcc lattice. ${ }^{[24]}$

Recently the grain boundary energies for pure $\alpha$-iron was computed by Kim et al. ${ }^{[14]}$ using molecular statics. In the current study, the supplemental online data in Reference 14 was used to plot the grain boundary energy distribution for different low CSL boundaries (Figure 11). The comparison between the grain boundary population and the relative grain boundary energy generally reveals an inverse correlation; i.e., the higher population the lower energy (Figures 6, 8, 9, and 11). For example, the $\Sigma 3$ boundary reveals maximum energy
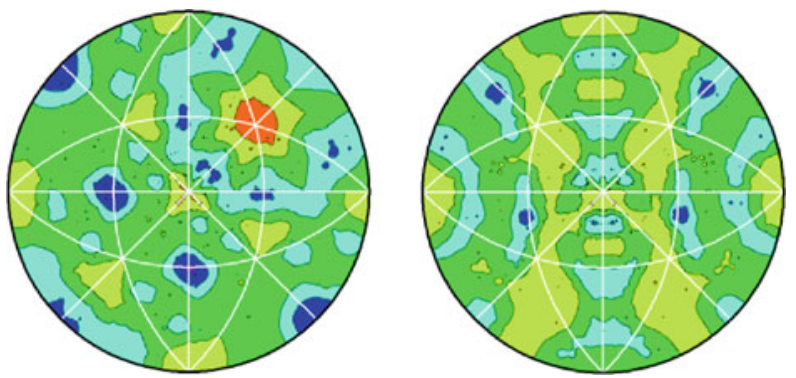

(a) $\sum 3=60^{\circ} /[111]$

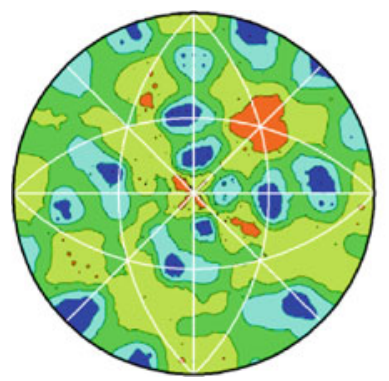

(b) $\sum 5=36.9^{\circ} /[100]$

(c) $\sum 7=38.2^{\circ} /[111]$

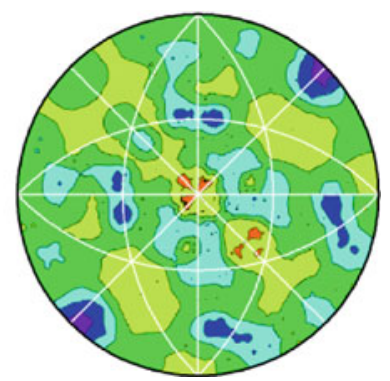

(d) $\sum 9=38.9^{\circ} /[110]$

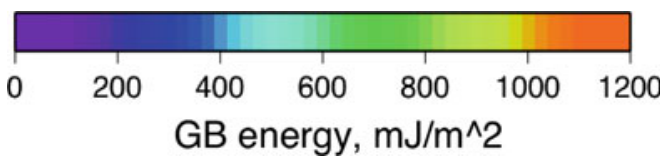

Fig. 11- The grain boundary energy distributions for different CSL boundaries using the computed grain boundary energies in Ref. [14]. 
Table I. Geometric Data for Selected CSL Boundaries

\begin{tabular}{lcccc}
\hline$\Delta g(\Sigma)$ & $n[u v w]$ & $d_{h k l}(\AA)$ & PCSD $\left(\right.$ Atoms $\left./ a^{2}\right)$ & $\lambda(\Delta g, n)(\mathrm{MRD})$ \\
\hline 3 & 111 & 0.58 & 1.15 & 0 \\
3 & 211 & 0.41 & 0.82 & 15.6 \\
5 & 100 & 0.50 & 2 & 0.88 \\
5 & 210 & 0.22 & 0.89 & 0.2 \\
5 & 310 & 0.16 & 0.63 & 0.3 \\
7 & 111 & 0.58 & 1.15 & 2.3 \\
7 & 321 & 0.13 & 0.53 & 1.2 \\
9 & 110 & 0.35 & 1.41 & 2.69 \\
9 & 221 & 0.17 & 0.67 & 0.6 \\
9 & 411 & 0.12 & 0.47 & 0.4 \\
11 & 110 & 0.35 & 1.41 & 1.5 \\
11 & 332 & 0.11 & 0.43 & 0.3 \\
11 & 311 & 0.30 & 0.60 & 0.3 \\
\hline
\end{tabular}

at the pure twist $\{111\}$ plane and minima along the zone of tilt boundaries, where they have the lowest and highest populations, respectively (Figures 6(f) and 11(a)). Similarly, the maximum and minimum energies for $\Sigma 9$ boundary were centered at the tilt and twist boundaries, respectively, which have opposite relationship with their populations (Figures 8(c) and 11(d)).

The grain boundary energy can be defined as the sum of the energy of two adjacent crystal surfaces minus the binding energy. ${ }^{[25]}$ The latter is related to the atoms bonded on either side of the boundary interface, which is closely related to the average interplanar spacing of two adjacent boundary surfaces. ${ }^{[26]}$ Therefore, it would be expected that the surfaces with low indices have low energy as they have high planar density with large interplanar spacing. However, the current result does not fully support this hypothesis, as there are some cases in which the low indices boundaries do not have necessarily low energy and consequently high population. For the case of the $\Sigma 3$ boundary, for example, the peak is centered on the symmetric tilt $\{112\}$ plane, which has lower interplanar spacing compared with the pure twist $\{111\}$ plane (i.e., low index), where the minimum distribution was located. This is consistent with experimental measurements ${ }^{[27]}$ and a recent simulation, ${ }^{[14]}$ shown in Figure 11(a), revealing that the $\{112\}$ twin grain boundary plane in bcc structure is lower in energy than the $\{111\}$ plane. To fully examine this hypothesis, the planar coincident site density (PCSD) was calculated using PCSD $=B /\left(h^{2}+k^{2}+l^{2}\right)^{1 / 2}$ for a number of low CSL grain boundary configurations and compared with their populations as shown in Table I and Figure 12. Here $B$ is the number of atoms per unit cell in the lattice, i.e., two for body centered cubic and four for face centered cubic materials. $h k l$ represent the grain boundary indices. The current result revealed that there is no strong correlation between the planar coincident site densities with the populations (Table I and Figure 12). For instance, the third highest population belongs to $\Sigma 7 /(111)$ with the eighth lowest planar coincidence site density (Figure 12). This can be related to the presence of (111) planes on either side of the interface similar to a coherent twin. In contrast, the symmetric $\Sigma 5$ tilt boundary, i.e., $37 \mathrm{deg} /[100]$, [100] reveals a relatively

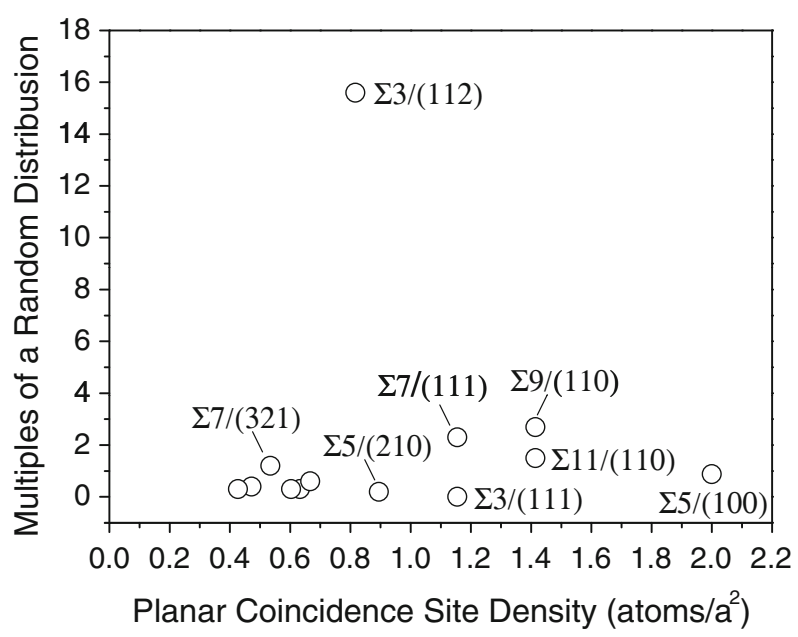

Fig. 12-Population of boundaries as a function of the PCSD for selected CSL boundaries listed in Table I.

low population, even though it has the highest planar coincidence site density among low CSL boundaries. The current observation, therefore, suggests that the planar coincidence cannot explain the grain boundary distribution/energy in IF steel. A similar conclusion was also made by others, studying grain boundaries in $\mathrm{Al}^{[9]}$ and gold. ${ }^{[28]^{7}}$

\section{SUMMARY}

In the present study, the grain boundary character distribution in a commercial IF steel was extensively examined as a function of lattice misorientation and boundary plane orientation. The grain boundaries were most frequently terminated on $\{111\}$ planes showing a tendency for low index planes with relatively low surface energy and large interplanar spacings. However, there were a few boundaries terminated on planes with higher index. For instance, the symmetric tilt boundaries comprised of two $\{112\}$ planes on either side of the interface dominated the population at the lattice misorientation of $60 \mathrm{deg}$ about the [111] axis. Interestingly, the measured grain boundary populations in the present study were inversely correlated to previously calculated grain boundary energies.

\section{ACKNOWLEDGMENTS}

The work at Deakin University was supported through the grants provided by the Australian Research Council. H. Beladi is also grateful to the financial assistant granted by Outside Study Program of Deakin University. The work at Carnegie Mellon University was supported by the MRSEC program of the National Science Foundation under Award Number DMR-0520425 and by the Office of Naval Research under grant N00014-11-1-0678. 


\section{REFERENCES}

1. M.D. Nave, M.R. Barnett, and H. Beladi: ISIJ Int., 2004, vol. 44, pp. $1072-78$.

2. E.O. Hall: Proc. Phys. Soc. Lond., 1951, vol. 64, pp. 747-53.

3. N.J. Petch: J. Iron Steel Inst., 1953, vol. 174, pp. 25-28.

4. R.W.K. Honeycombe and H.K.D.H. Bhadeshia: Steels: Microstructures and Properties, 2nd ed., Arnold, London, 1995.

5. N. Tsuji, Y. Ito, Y. Saito, and Y. Minamino: Scripta Mater., 2002, vol. 47, pp. 893-99.

6. G.S. Rohrer, D.M. Saylor, B. El Dasher, B.L. Adams, A.D Rollett, and P. Wynblatt: Z. Metallkd., 2004, vol. 95, pp. 197-214.

7. S.J. Dillon and G.S. Rohrer: J. Am. Ceram. Soc., 2009, vol. 92, pp. $1580-85$.

8. J. Li, S.J. Dillon, and G.S. Rohrer: Acta Mater., 2009, vol. 57, pp. 4304-11.

9. D.M. Saylor, B.S. El Dasher, A.D. Rollett, and G.S. Rohrer: Acta Mater., 2004, vol. 52, pp. 3649-55.

10. D.M. Saylor, B.S. El Dasher, T. Sano, and G.S. Rohrer: J. Am. Ceram. Soc., 2004, vol. 87, pp. 670-76.

11. D.M. Saylor, B.S. El Dasher, Y. Pang, H.M. Miller, P. Wynblatt, A.D. Rollett, and G.S. Rohrer: J. Am. Ceram. Soc., 2004, vol. 87, pp. 724-26.

12. D.M. Saylor, A. Morawiec, and G.S. Rohrer: Acta Mater., 2003, vol. 51 , pp. $3675-86$

13. T.A. Bennett, C.S. Kim, G.S. Rohrer, and A.D. Rollett: Mater. Sci. Forum, 2004, vols. 467-470, pp. 1057-62.
14. H.K. Kim, W.S. Ko, H.J. Lee, S.G. Kim, and B.J. Lee: Scripta Mater., 2011, vol. 64, pp. 1152-55.

15. A. Morawiec, J.A. Szpunar, and D.C. Hinz: Acta Metall. Mater., 1993, vol. 41, pp. 2825-32.

16. H. Beladi, P. Cizek, and P.D. Hodgson: Metall. Mater. Trans. A, 2009, vol. 40A, pp. 1175-89.

17. V. Randle, G.S. Rohrer, H.M. Miller, M. Coleman, and G.T. Owen: Acta Mater., 2008, vol. 56, pp. 2363-73.

18. A. Morawiec: J. Appl. Crystallogr., 2011, vol. 44, pp. 1152-55.

19. J.P. Hirth and J. Lothe: Theory of Dislocations, 2nd ed., Krieger Publishing Company, Florida, 1982, pp. 274-366.

20. D.A. Curry: Metal Sci., 1980, vol. 14, pp. 319-26.

21. B. Gale, R.A. Hunt, and M. McLean: Philos. Mag., 1972, vol. 25, pp. $947-60$

22. P.B. Mee: Trans. Metall. Soc. AIME, 1968, vol. 242, pp. $2155-$ 61.

23. G.S. Rohrer, V. Randle, C.S. Kim, and Y. Hu: Acta Mater., 2006, vol. 54 , pp. 4489-4502.

24. D. Wolf and S. Phillpot: Mater. Sci. Eng. A, 1989, vol. 107, pp. 3-14

25. D. Wolf: J. Mater. Res., 1990, vol. 5, pp. 1708-30.

26. A.P. Sutton: Prog. Mater Sci., 1992, vol. 36, pp. 167-202.

27. C.G. Dunn, F.W. Daniels, and M.J. Bolton: J. Met. Trans. AIME, 1950, vol. 188, pp. 368-69.

28. P.J. Goodhew, T.Y. Tan, and R.W. Balluffi: Acta Metall., 1978, vol. 35 , pp. 557-67. 